\title{
Some consequences of stress range dependent constitutive models in creep
}

\author{
JT Boyle \\ Department of Mechanical \& Aerospace Engineering, University of Strathclyde, Glasgow, Scotland \\ Submitted to the IUTAM Symposium Advanced Materials Modelling for Structures
}

April $23^{\text {rd }}-27^{\text {th }}, 2012$, Ecole des Mines de Paris, France

Studies of the behaviour of structural components at high temperature subject to creep have a long history and their behavioural characteristics, certainly for constant and cyclic load, well established. However, the majority of these studies have been based on relatively simple constitutive models in particular time- or strain-hardening for primary creep with a basic power-law model for secondary creep. These basic models can be enhanced to include tertiary creep with the introduction of wellknown damage models of the Kachanov-Rabotnov type. Over the past decade the modelling of advanced high temperature materials has improved considerably and, coupled with advances in computational modelling of real-world components, has allowed a much more realistic and detailed representation of structural behaviour at high temperature subject to precise thermo-mechanical load histories. Nevertheless, most design rules for high temperature have been developed based on an understanding of creep behaviour with the simple constitutive models, which remain the most widely used even in design and assessment approaches using detailed nonlinear finite element analysis. To be useful for design purposes, modern advanced material models need to be able to make reference to conventional understanding of the behavioural characteristics of structures subject to creep. Yet this can often be problematic, not due to imprecision in the advanced models, but rather deficiencies in the simple models. Recent studies (by the author and others) of the behaviour of structures using simple creep models, but ones which are stress range dependent (that is, the form of the constitutive model, even for secondary creep, changes as the stress increases from low through moderate to high stress) have shown that under some types of loading creep behaviour of structures in significantly altered, while for others familiar design concepts can remain valid. Simple stress range dependent constitutive models have been around for well over 60 years but have been rarely used: the power-law has been favoured since it is simple to use and has some desirable features which have led to robust simplified methods for creep design. The aim of this paper is to examine further the consequences of using a stress range dependent constitutive model in structural design and analysis. Several simple structural components under different loading conditions will be examined and the results compared to current design practice. 